\title{
The role of the ICOS-B7h $T$ cell costimulatory pathway in transplantation immunity
}

\author{
Hiroshi Harada, ${ }^{1}$ Alan D. Salama, ${ }^{1,2}$ Masayuki Sho, ${ }^{1}$ Atsushi Izawa, ${ }^{1}$ Sigrid E. Sandner, ${ }^{1}$ \\ Toshiro Ito, ${ }^{2}$ Hisaya Akiba, ${ }^{3}$ Hideo Yagita, ${ }^{3}$ Arlene H. Sharpe, ${ }^{4}$ Gordon J. Freeman, ${ }^{5}$ \\ and Mohamed H. Sayegh ${ }^{1,2}$
}
${ }^{1}$ Division of Nephrology, Department of Medicine, Children's Hospital and Harvard Medical School, Boston, Massachusetts, USA
${ }^{2}$ Laboratory of Immunogenetics and Transplantation, Brigham and Women's Hospital, Boston, Massachusetts, USA
${ }^{3}$ Department of Immunology, Juntendo University School of Medicine, Tokyo, Japan
${ }^{4}$ Department of Pathology, Brigham and Women's Hospital, Boston, Massachusetts, USA
${ }^{5}$ Department of Medical Oncology, Dana-Farber Cancer Institute, Boston, Massachusetts, USA

\begin{abstract}
Inducible costimulatory molecule (ICOS) plays a pivotal role in $\mathrm{T}$ cell activation and Th1/Th2 differentiation. ICOS blockade has disparate effects on immune responses depending on the timing of blockade. Its role in transplantation immunity, however, remains incompletely defined. We used a vascularized mouse cardiac allograft model to explore the role of ICOS signaling at different time points after transplantation, targeting immune initiation (early blockade) or the immune effector phase (delayed blockade). In major histocompatibility-mismatched recipients, ICOS blockade prolonged allograft survival using both protocols but did so more effectively in the delayed-treatment group. By contrast, in minor histocompatibility-mismatched recipients, early blockade accelerated rejection and delayed blockade prolonged graft survival. Alloreactive $\mathrm{CD}^{+} \mathrm{T}$ cell expansion and alloantibody production were suppressed in both treatment groups, whereas only delayed blockade resulted in suppression of effector $\mathrm{CD8}^{+} \mathrm{T}$ cell generation. After delayed ICOS blockade, there was a diminished frequency of allospecific IL-10-producing cells and an increased frequency of both IFN- $\gamma$ - and IL-4-producing cells. The beneficial effects of ICOS blockade in regulating allograft rejection were seen in the absence of $\mathrm{CD} 28$ costimulation but required $\mathrm{CD}^{+}$cells, cytotoxic T lymphocyte antigen-4, and an intact signal transducer and activator of transcription- 6 pathway. These data define the complex functions of the ICOS-B7h pathway in regulating alloimmune responses in vivo.
\end{abstract}

J. Clin. Invest. 112:234-243 (2003). doi:10.1172/JCI200317008.

\section{Introduction}

An efficient $T$ cell response requires costimulatory signals delivered by APCs in addition to specific antigen signals acting on the $\mathrm{T}$ cell receptor (TCR). The prototypical costimulatory molecule $\mathrm{CD} 28$ provides a strong mitogenic signal, induces a large array of effector cytokines, and upregulates cell survival genes such as $\mathrm{Bcl}-\mathrm{x}_{\mathrm{L}}(1-3)$. The CD40-CD154 pathway is another key pathway for $\mathrm{T}$ cell activation (4-6). However, it is apparent that $\mathrm{T}$ cell activation and transplant rejection can proceed in the

Received for publication September 30, 2002, and accepted in revised form April 22, 2003.

Address correspondence to: Mohamed H. Sayegh, Laboratory of Immunogenetics and Transplantation, Brigham and Women's Hospital, 75 Francis Street, Boston, Massachusetts 02115, USA. Phone: (617) 732-5259; Fax: (617) 732-5254;

E-mail: msayegh@rics.bwh.harvard.edu.

Alan D. Salama, Masayuki Sho, and Atsushi Izawa contributed equally to this work.

Conflict of interest: The authors have declared that no conflict of interest exists.

Nonstandard abbreviations used: inducible costimulatory molecule (ICOS); T cell receptor (TCR); cytotoxic T lymphocyte antigen-4 (CTLA-4); signal transducer and activator of transcription$4^{-/-}\left(\right.$STAT4 $\left.^{-/-}\right)$; median survival time (MST); PBS containing $0.05 \%$ Tween (PBST); anti-bm12 (ABM); experimental autoimmune encephalomyelitis (EAE). absence of CD28-B7 or CD40-CD154 signals, since mice lacking CD28 are able to acutely reject cardiac allografts $(7,8)$, and CD154-deficient mice go on to develop chronic rejection (9). In addition, memory $T$ cells $(10,11)$ and $\mathrm{CD}^{+} \mathrm{T}$ cells (12), both important mediators of allograft loss, are far less dependent on these costimulatory signals than naive $\mathrm{T}$ cells, making them less susceptible to costimulatory blocking strategies.

Inducible costimulatory molecule (ICOS), another member of the $\mathrm{CD} 28$ superfamily, has unique roles in $\mathrm{T}$ cell activation and differentiation (13-15), splenic germinal center formation, and immunoglobulin class switching $(14,16,17)$. ICOS is inducible within 48 hours of $\mathrm{T}$ cell activation on both $\mathrm{CD}^{+}$and $\mathrm{CD}^{+}$cells (13) after CD28 signaling (18), whereas cytotoxic T lymphocyte antigen-4 (CTLA-4) ligation prevents its upregulation (19). ICOS binds to its ligand B7h (20-24), which is constitutively expressed at low levels on the surface of APCs and is upregulated by TNF- $\alpha$ or lipopolysaccharide (25). ICOS regulates both Th1 and Th 2 cell differentiation (3, $15,18,26-31)$. Recent reports have suggested that CD28 and ICOS play disparate roles in $\mathrm{T}$ cell differentiation, $\mathrm{CD} 28$ signaling being responsible for $\mathrm{T}$ cell priming and ICOS signaling regulating effector responses $(3,32,33)$. In a model of autoimmune encephalomyelitis, ICOS 
blockade during immune priming exacerbates the disease, but blockade during the effector phase ameliorates it (33). The precise reason for this disparity is unclear, but it is known that ICOS-B7h signal blockade during antigen priming impairs Th2 development and may thus lead to Th1 polarization, hence exacerbating autoimmune disease $(33,34)$ as well as acute graft-versus-host disease (35). These observations are supported by the findings of lethal autoimmune disease that develops in ICOS-deficient mice (15). In transplantation models, ICOS expression was found to be upregulated in cardiac allografts during acute rejection, and ICOS-B7h pathway blockade produced a modest but significant prolongation of graft survival (30). However, the effect of ICOS blockade during different phases of the alloimmune response was not addressed. Such understanding is of considerable importance for the potential translation of ICOS blockade strategies to a clinical setting. In this study, we report on the role of ICOS-B7h signaling at different time points in the alloimmune response in vivo and its unique mechanism controlling alloimmune responses in vivo.

\section{Methods}

Animals. C57BL/6 $\left(\mathrm{H}-2^{\mathrm{b}}\right)$ and $\mathrm{BALB} / \mathrm{c}\left(\mathrm{H}-2^{\mathrm{d}}\right)$ mice were purchased from Taconic Farms (Germantown, New York, USA). WT 129S1/SvImJ (H-2 $\left.{ }^{\mathrm{b}}\right)$ and B10.D2 (H-2 ${ }^{\mathrm{d}}$ ) mice were from the Jackson Laboratory (Bar Harbor, Maine, USA). ICOS-deficient (ICOS $\left.{ }^{-/}\right)$129S4/SvJae (H-2 ${ }^{\text {b }}$ mice $(14,29)$ were maintained in accordance with the institutional guidelines of the Brigham and Women's Hospital and Harvard Medical School. BALB/c background signal

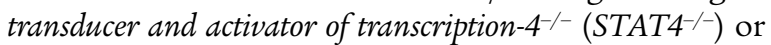
$S T A T 6^{-1-}$ mice were purchased from the Jackson Laboratory. Animals were used at 6-10 weeks of age.

Reagents and antibodies. The anti-ICOS mAb 7E.17G9 (rat IgG2b isotype)(18), anti-B7h mAb HK5.3 (rat IgG2a isotype) (36), and anti-CTLA-4 mAb 4F10 (a kind gift of J. Bluestone) (37) have all been described previously. These antibodies were produced by Bioexpress Cell Culture Services (West Lebanon, New Hampshire, USA). Anti-CD8-depleting $\mathrm{mAbs}$ were prepared from hybridoma 2.43 (rat anti-mouse CD8) obtained from American Type Culture Collection (Manassas, Virginia, USA).

Cardiac transplantation and treatment protocols. BALB/c mice (WT, STAT4 ${ }^{--}$and STAT6 ${ }^{--}$) were used as recipients and C57BL/6 or B10.D2 mice as donors. 129S1/SvImJ WT mice and $129 \mathrm{~S} 4 / \mathrm{SvJae} \mathrm{ICOS}^{-/-}$mice were used as recipients and fully allogeneic BALB/c WT mice as donors in some experiments. Vascularized heart grafts were transplanted using microsurgical techniques as described by Corry et al. (38). Briefly, donor and recipient mice were anesthetized with pentobarbital. Donor hearts were harvested and placed in chilled physiological saline, during which time the recipient mice were prepared. The donor heart was anastomosed to the recipient abdominal aorta and inferior vena cava using microsurgical techniques. Graft function was assessed by daily palpation of the abdomen. Rejection was defined as complete cessation of cardiac contractility as determined by direct visualization. Loss of graft function within 48 hours after transplantation was considered a technical failure (less than $5 \%$ on average), and these animals were excluded from further analysis. Graft survival is shown as the median survival time (MST) in days. Recipients received anti-ICOS $\mathrm{mAb}$ or anti-B7h $\mathrm{mAb}$ (early treatment protocol, $500 \mu \mathrm{g}$ intraperitoneally on day 0 and $250 \mu \mathrm{g}$ intraperitoneally on days 2,4 , and 6 ; delayed treatment protocol, $500 \mu \mathrm{g}$ intraperitoneally on day 4 and $250 \mu \mathrm{g}$ intraperitoneally on days 6,8 , and 10$)$. In some experiments, anti-CTLA- 4 mAb was administered (500 $\mu \mathrm{g}$ intraperitoneally on day 0 and $250 \mu \mathrm{g}$ intraperitoneally on days 2,4 , and 6 ) alone or in conjunction with antiICOS mAb using early or delayed treatment protocols. $\mathrm{CD}^{+} \mathrm{T}$ cell depletion was achieved by treating mice preoperatively with $100 \mu \mathrm{l}$ of ascites containing anti-CD8depleting $\mathrm{mAb}$ (roughly equivalent to $100 \mu \mathrm{g}$ of purified antibody) on days 6, 3, and 1 before transplantation (8). This regimen ensures over $95 \%$ depletion of $\mathrm{CD}^{+}$cells in the peripheral blood on the day of transplantation.

ELISPOT assay. The technique for ELISPOT analysis has been described recently by our group and others (8, 39-41). Immunospot plates (Cellular Technology Ltd., Cleveland, Ohio, USA) were coated with $4 \mu \mathrm{g} / \mathrm{ml}$ of rat anti-mouse IFN- $\gamma$ mAb (R4-6A2), rat anti-mouse IL-4 mAb (BVD4-1D11), or rat anti-mouse IL-10 mAb (JES52A5)(all from Pharmingen, San Diego, California, USA) in sterile PBS overnight. The plates were then blocked for 1 hour with sterile PBS containing 1\% BSA-fraction $V$ and washed three times with sterile PBS. Splenocytes $\left(10^{6}\right.$ in $200 \mu \mathrm{l}$ of HL-1 medium containing $1 \%$ L-glutamine) were then placed in each well in the presence of $10^{6}$ irradiated (30 Gy) syngeneic or allogeneic splenocytes and cultured for 24 hours at $37^{\circ} \mathrm{C}$ in $5 \% \mathrm{CO}_{2}$. After washing with PBS followed by washing with PBS containing $0.05 \%$ Tween (PBST), $2 \mu \mathrm{g} / \mathrm{ml}$ of biotinylated rat anti-mouse IFN- $\gamma$ detection mAb (XMG1.2) or $4 \mu \mathrm{g} / \mathrm{ml}$ of biotinylated anti-mouse IL-4 mAb (BVD6-24G2) or IL-10 mAb (SXC-1) (all from Pharmingen) were added overnight. The plates were then washed four times in PBST, followed by 2 hours of incubation with horseradish peroxidase-conjugated streptavidin (Dako, Carpenteria, California, USA) diluted at 1:2000 in PBS/1\% BSA. After washing three times with PBST followed by PBS, the plates were developed using 3-amino-9-ethylcarbazole (Sigma-Aldrich, St. Louis, Missouri, USA). The resulting spots were counted on a computer-assisted enzyme-linked immunospot image analyzer (Cellular Technology Ltd.), and frequencies were expressed as the number of cytokine-producing spots per million splenocytes. In additional experiments, splenocytes were depleted of $\mathrm{CD}^{+}$cells by incubating splenocyte preparations with anti-CD8 $\mathrm{mAb}$ (clone 53-6.7, Pharmingen) followed by magnetic bead depletion (Dynal, Oslo, Norway), as per the manufacturer's instructions.

$C D 8^{+}$effector $T$ cell enumeration. Recipient splenocytes were isolated 10 days after transplantation, and red cells were lysed with AKLysis buffer (Biowhittaker, Walkersville, Maryland, USA). Cells $\left(10^{6}\right)$ were 
stained with anti-CD8-FITC, anti-CD62L-APC, and anti-CD44-PE (all from Pharmingen). Flow cytometry was performed using a FACSCalibur flow cytometer system (Beckton Dickinson, San Jose, California, USA) and analyzed using CellQuest software (Becton Dickinson). Percentages of effector $\mathrm{CD}^{+}$cells expressing the $\mathrm{CD} 44^{\text {high }} \mathrm{CD} 62 \mathrm{~L}^{\text {low }}$ phenotype were measured in triplicate, as previously described (42). Results are representative of three experiments.

Measurement of serum alloantibody. Naive splenocytes $\left(10^{6}\right)$ of donor strain C57BL/6 were incubated for 30 minutes at $4{ }^{\circ} \mathrm{C}$ with $50 \mu \mathrm{l}$ of serially diluted sera obtained from naive BALB/c or C57BL/ 6 mice (controls), C57BL/6 heart recipients on days 10-14 after transplantation with no treatment, and those treated with either early or delayed anti-ICOS mAb. Cells were washed twice, incubated with $50 \mu \mathrm{l}$ of FITC-conjugated anti-mouse IgG1 or anti-mouse IgG2a (both from Pharmingen) at $4^{\circ} \mathrm{C}$ for 30 minutes, and analyzed by flow cytometry using a FACSCalibur (Becton Dickinson) and CellQuest software (Becton Dickinson). The percentage of donor cells stained at each serum dilution and the relative median fluorescence was determined (43) and compared with that of control samples.

Adoptive transfer of $\mathrm{CD}^{+} \mathrm{TCR}$ transgenic $\mathrm{T}$ cells. WT or nude C57BL/6 mice were adoptively transferred with 2-3 million $\mathrm{CD}^{+} \mathrm{T}$ cells from anti-bm $12(\mathrm{ABM})$ mice carrying a TCR transgene reactive to a mutant class II MHC, $\mathrm{I}_{-} \mathrm{A}^{\mathrm{bm} 12}$, expressed in bm12 mice (44-46). Recipients were then transplanted with bm12 skin grafts as described $(45,46)$. In this model, the alloreactive $\mathrm{CD}^{+} \mathrm{T}$ cells can be tracked using specific mAbs to the transgenic TCR $V \alpha 2$ and $V \beta 8$ chains (46). While some transplanted mice were left untreated, others received anti-ICOS mAb according to either the early- or delayed-treatment protocols described above. Ten days after transplantation, the draining lymph nodes were removed and stained with anti-CD4-PerCP, anti-V $\alpha 2.1$-FITC and anti-V $\beta 8.1$ biotin, followed by streptavidin-APC as well as annexin V-PE (all from Pharmingen) to quantitate in vivo alloreactive $T$ cell expansion and apoptosis. Cells were analyzed by flow cytometry using a FACSCalibur (Becton Dickinson) and CellQuest software (Becton Dickinson).

Histology. Allogeneic heart grafts were harvested 10 days after transplantation. All specimens were fixed in $10 \%$ buffered formalin and embedded in paraffin. Coronal sections were cut and stained with hematoxylin and eosin. Light microscopy was performed to assess overall cellularity and preservation of myocardial tissue.

Statistics. To calculate graft survival, Kaplan-Meier survival graphs were constructed, and the log-rank comparison of the groups was used to calculate $P$ values. Significant differences between experimental groups in the ELISPOT assay, transgenic $\mathrm{CD} 4^{+} \mathrm{T}$ cell model, effector $\mathrm{CD}^{+} \mathrm{T}$ cell generation assay, and alloantibody production assay were analyzed using Student's $t$ test. Differences were considered to be significant when $P$ was less than 0.05 .

\section{Results}

ICOS-B7h signal blockade with anti-ICOS or anti-B7h $m A b$ prolongs cardiac allograft survival. We used a previously described blocking anti-ICOS mAb (18) and anti-B7h $\mathrm{mAb}(36)$ to study the role of ICOS-B7h inactivation in vascularized cardiac allograft rejection. Cardiac grafts from $\mathrm{C} 57 \mathrm{BL} / 6$ donors $\left(\mathrm{H}-2^{\mathrm{b}}\right)$ were transplanted into fully allogeneic BALB/c $\left(\mathrm{H}-2^{\mathrm{d}}\right)$ recipients. Untreated recipients acutely rejected their grafts between 8 and 12
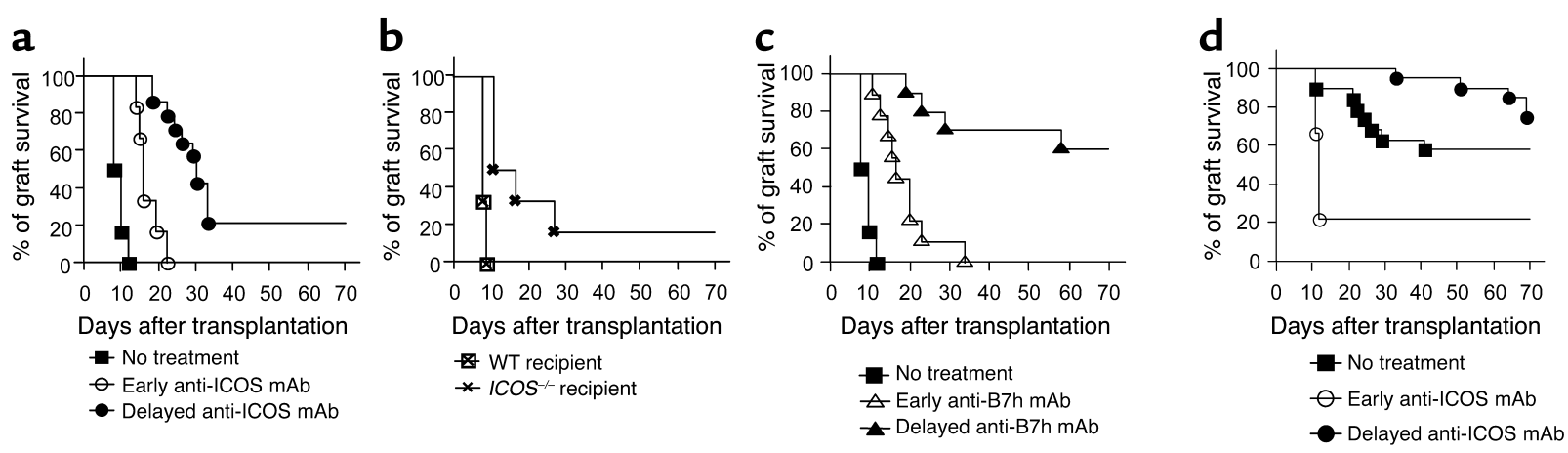

\section{Figure 1}

Delayed ICOS-B7h signal blockade more effectively prolongs allograft survival. (a) Vascularized C57BL/6 ( $\left.\mathrm{H}-2^{\mathrm{b}}\right)$ hearts were transplanted into $\mathrm{BALB} / \mathrm{c}\left(\mathrm{H}-2^{\mathrm{d}}\right)$ recipients and treated with anti-ICOS mAb using two different protocols. Early blockade prolonged graft survival (MST, 16 days; $n=6 ; P<0.0001$ ) versus untreated control group (MST, 9 days; $n=6$ ). However, delayed treatment prolonged graft survival more than early treatment (MST, 30 days; $n=14 ; P<0.0005$ versus early treatment group). (b) Vascularized BALB/c hearts were transplanted into $129 \mathrm{~S} 1 / \mathrm{SvlmJ}$ WT mice or $129 \mathrm{~S} 4 / \mathrm{SvJae}_{\text {ICOS }}{ }^{-1-}$ mice. Allograft survival in $/ \mathrm{COS}^{-/-}$recipients was significantly prolonged (MST, 14 days; $n=6 ; P<0.001)$ versus that in WT (MST, 8 days; $n=6)$. (c) Transplant recipients treated with anti-B7h mAb using the same two protocols also demonstrated that early blockade significantly prolonged graft survival (MST, 20 days; $n=9 ; P=0.0001$ versus control), while delayed treatment prolonged graft survival even further (MST, $>70$ days; $n=10 ; P<0.005$ versus the early treatment group). (d) Vascularized B10.D2 $\left(\mathrm{H}-2^{d}\right)$ hearts were transplanted into BALB/c $\left(\mathrm{H}-2^{\mathrm{d}}\right)$ recipients and treated with anti-ICOS mAb according to the above protocols. Control grafts were rejected with an MST of 26 days. While delayed blockade prolonged graft survival (MST, 100 days; $P=0.049$ versus control), early blockade resulted in a trend to accelerated graft rejection (MST, 12 days; $P=0.0008$ versus delayed blockade; $P=$ not statistically significant versus control). 
days with an MST of 9 days. Early (days 0, 2, 4, and 6) anti-ICOS $\mathrm{mAb}$ treatment significantly extended graft survival (MST, 16 days), and delayed (days 4, 6, 8, and 10) treatment further prolonged allograft survival (MST, 30 days), with $20 \%$ of grafts surviving long-term (more than 60 days) (Figure 1a). Importantly, it was the delay in starting therapy rather than the duration of therapy that was critical, since administration of antiICOS mAb from day 0 to day 10 every other day did not result in improved outcome as compared with early anti-ICOS mAb treatment (MST, 18 days; $n=4$; not statistically significant versus the early-treatment group).

We then compared allograft survival in WT and ICOS $^{-/-}$(129 background) recipients (14). ICOS IC $^{-/}$recipients had prolonged cardiac allograft survival (BALB/c donors) of a magnitude similar to that of the early antiICOS mAb treatment group (MST, 14 days) (Figure 1b).

Similarly but more effectively, using the anti-B7h $\mathrm{mAb}$ to target ICOS ligand, early blockade extended graft survival (MST, 20 days), and delayed treatment promoted even greater prolongation of allograft survival (MST, more than 70 days), with $60 \%$ of recipients exhibiting long-term graft survival (Figure 1c). This highlights the universal effect of the delayed blockade of the ICOS-B7h signaling pathway in vivo.

Furthermore, histological assessment of rejected grafts mirrored the survival data. In untreated control recipients (Figure 2a), diffuse mononuclear cell infiltration with associated myocyte necrosis and interstitial hemorrhage was seen. In the anti-ICOS mAb earlytreatment group, there was scattered inflammatory cell infiltration within the grafts with relatively preserved myocytes (Figure $2 \mathrm{~b}$ ), whereas in the delayed-treatment group little inflammatory infiltrate was seen, and myocytes were undamaged (Figure2c).

These findings are in contrast to some of the reported results in the experimental autoimmune encephalomyelitis (EAE) model, in which ICOS deficiency and early ICOS blockade exacerbated disease, but late blockade ameliorated it $(33,34)$. One important difference between auto- and alloimmune responses is the frequency of responding antigen-specific $\mathrm{T}$ cells, with significantly higher frequencies of alloreactive $\mathrm{T}$ cells (47). Therefore, in order to investigate whether alloreactive $\mathrm{T}$ cell clone size was important in determining the outcome of ICOS blockade in vivo, we transplanted minor-mismatched B10.D2 cardiac allografts into BALB/c recipients and blocked the ICOS pathway with anti-ICOS $\mathrm{mAb}$. We recently reported that the frequency of alloreactive $T$ cells is $5-10$ times lower in the same strains of minor versus major-mismatched allograft recipients (48). Although untreated recipients rejected their grafts with an MST of 26 days, delayed ICOS blockade again resulted in significantly prolonged graft survival, with an MST of 100 days $(P=0.049$ as compared with controls and 0.0008 as compared with early blockade). Interestingly, early blockade was associated with decreased graft survival (MST, 12 days), although this was not statistically different from untreated controls (Figure 1d). This somewhat unusual result, in which an immunomodulatory strategy is less effective in minor-mismatched versus major-mismatched combinations, demonstrates that the alloreactive $\mathrm{T}$ cell clone size has a great effect in determining the outcome of ICOS-B7h blockade.

Role of ICOS-B7b in alloreactive $C D 4^{+} T$ cell expansion in vivo. ICOS has been shown to regulate antigen-specific $\mathrm{CD}^{+}$ $T$ cell activation and cytokine production (18). To investigate the role of ICOS-B7h signaling in expansion of alloreactive $\mathrm{CD}^{+} \mathrm{T}$ cells in vivo, we made use of a newly developed CD4-dependent alloreactive TCR transgenic model (44-46). This model allowed us to track the expansion of alloantigen-specific $\mathrm{CD} 4^{+} \mathrm{T}$ cells in vivo during the process of allograft rejection, and we have recently described the kinetics of such expansion (46). Ten days after transplantation, both early and delayed ICOS blockade resulted in a significant reduction (54\% and 53\%, respectively) in expansion of alloantigen-specific $\mathrm{CD}^{+} \mathrm{T}$ cells $(P<0.0001$ for either as compared with untreated transplant controls) (Figure 3a). Moreover, this reduction was not due to increased $\mathrm{CD}^{+} \mathrm{T}$ cell apoptosis, since there was no difference in the percentage of annexin $\mathrm{V}$ stained cells between the groups (Figure $3 \mathrm{~b}$ ).

Effect of early and delayed ICOS blockade on alloantigen-specific cytokine production. Since differences in IFN- $\gamma$ production after early and delayed ICOS blockade were previously described in experimental autoimmune models (33), and since ICOS is known to influence Th1 and Th2 cell differentiation (28), we studied alloantigen-specific Th1 (IFN- $\gamma$ ) and Th2 (IL-4 and IL-10) cytokine production in recipients treated with early or delayed ICOS blockade, using a previously published ELISPOT assay (40). Recipient splenocytes were collected 10-14 days after transplantation, and the frequency of IFN- $\gamma-$, IL-4- and IL-10-producing allospecific cells was measured. In the delayed- but not the early-treatment group, the frequency of alloreactive IFN- $\gamma$-producing splenocytes was increased as compared with the untreated control recipients $(P=0.0115$ as compared with control) (Figure 4a). By contrast, both early and delayed blockade

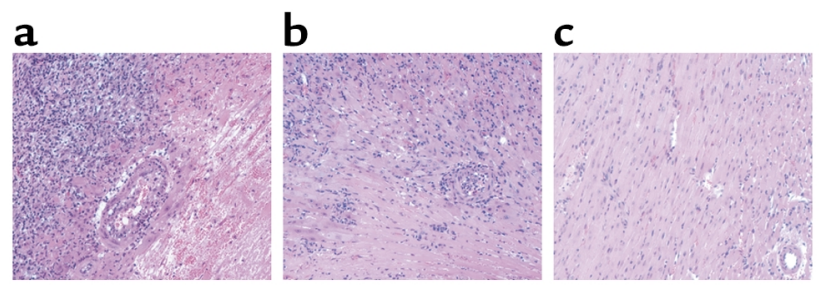

\section{Figure 2}

Histology of murine cardiac allografts from animals treated with ICOS-B7h signal blockade. C57BL/ 6 grafts were harvested from BALB/c recipients 10 days after cardiac transplantation in animals receiving no treatment and either early or delayed anti-ICOS mAb therapy. Rejected grafts from untreated control recipients demonstrated diffuse mononuclear cell infiltration, myocyte destruction, and interstitial hemorrhage (a). There was a scattered inflammatory cell infiltrate in the grafts treated with the early ICOS-B7h blockade (b), whereas few inflammatory cells and preserved cardiac myocytes were seen in the grafts treated with delayed therapy (c). Magnification, $\times 400$ (H\&E). 

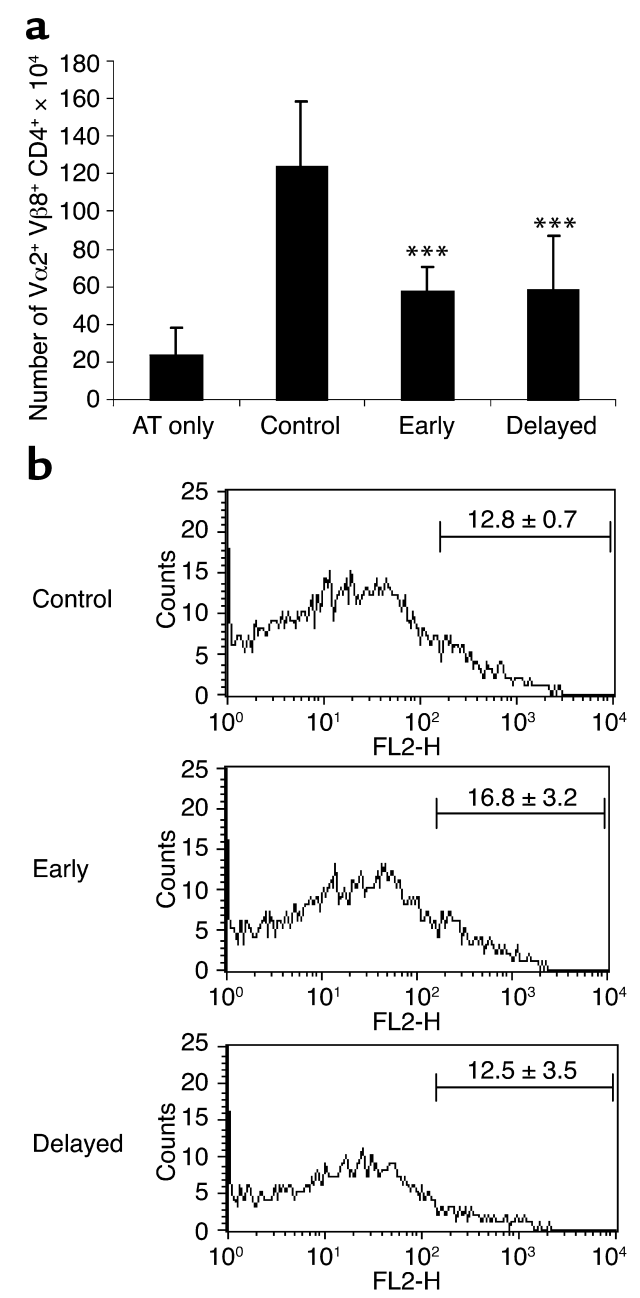

\section{Figure 3}

Expansion and apoptosis of alloreactive CD4 ${ }^{+} \mathrm{T}$ cells after ICOS-B7h blockade. After adoptive transfer of transgenic ABM CD4 ${ }^{+} \mathrm{T}$ cells into nude mice, the animals were transplanted with bm12 skin and left untreated or were given anti-ICOS mAb according to the two protocols. Ten days later, draining lymph nodes were removed and the number of alloreactive $C D 4^{+} T$ cells was examined $(\mathbf{a})$. To control for homeostatic proliferation, some animals were adoptively transferred with cells but received no transplant (AT only), and these demonstrated only modest proliferation. By comparison, there was a marked expansion of $\mathrm{CD}^{+} \mathrm{T}$ cells after transplantation, which was significantly reduced after early and delayed ICOS-B7h blockade (by $54 \%$ and $53 \%$, respectively; ${ }^{* *} P<0.0001$ for either as compared with transplant controls). (b) By gating on the transgenic CD4+ $\mathrm{T}$ cells, the percentage of alloreactive cells undergoing apoptosis was measured by annexin $\mathrm{V}$ staining. There was no difference in the percentage of cells undergoing apoptosis between controls and early or delayed blockade, demonstrating that the reduction in expansion after ICOS-B7h blockade was not simply due to increased apoptosis. FL2-H, annexin $\mathrm{V}$.

significantly decreased the frequency of IL-10-producing cells $(P=0.0047$ for both early and delayed treatment as compared with controls) (Figure 4b). Interestingly, early blockade produced a slight but nonsignificant increase in the frequency of IL-4-producing cells as compared with controls, whereas delayed blockade significantly increased the IL-4-producing $\mathrm{T}$ cell frequency
$(P=0.0006$ as compared with controls) (Figure 4c). Furthermore, after ex vivo depletion of $\mathrm{CD}^{+} \mathrm{T}$ cells from splenocyte preparations, IL-4 production was diminished in both control and delayed ICOS blockade groups, although only the decline in the delayed ICOS blockade group was significantly greater than in controls $(P=0.035$ for CD8-depleted ICOS blockade recipients as compared with CD8-depleted control recipients) (Figure 4d). These data demonstrate that in the context of alloimmune responses, delayed ICOS blockade significantly augments IFN- $\gamma$ and IL-4 production, while diminishing IL-10 production, and that induction of IL-4 production is dependent on a subset of alloreactive $\mathrm{CD}^{+} \mathrm{T}$ cells.

We then investigated the role of the ICOS-B7h pathway in alloreactive Th1/Th2 cell differentiation by using STAT4- or STAT6-deficient animals with polarized T helper cell environments. Consistent with previous reports (40), both $S T A T 4^{-/-}$(Th1-defective) and STAT6 $^{-/-}$(Th2-defective) recipients were able to reject allografts with a tempo similar to that of WT recipients (MST, 10 days for both groups). In a similar fashion to WT recipients, delayed treatment with anti-ICOS $\mathrm{mAb}$ resulted in significant prolongation of allograft survival in STAT4 $4^{--}$animals (MST, 24 days) (Figure 5a). By contrast, in STAT6 ${ }^{-/-}$mice, delayed anti-ICOS mAb did not extend allograft survival at all as compared with the untreated control group (Figure $5 \mathrm{~b}$ ). This is not due to an inherent resistance of $S T A T 6^{-/-}$recipients to $\mathrm{T}$ cell costimulatory blockade, since CTLA-4 Ig prolonged cardiac allograft survival in these recipients (41). These data suggest that prolongation of allograft survival by ICOS blockade requires an intact STAT6 pathway.

The role of ICOS in generation of alloreactive effector $C D 8^{+}$ Tcells in vivo. The outcome of ICOS signaling on $\mathrm{CD}^{+}$ $T$ cell function is unclear $(31,49-51)$. In order to assess the effect of ICOS-B7h blockade on alloreactive $\mathrm{CD}^{+}$ $\mathrm{T}$ cells, we measured the percentage of effector $\mathrm{CD}^{+}$ cells (expressing a CD62L Low $C D 44^{\text {high }}$ phenotype) generated 10 days after transplantation, in untreated control recipients and in recipients treated with early or delayed ICOS blockade. A significant decrease (34\%) in the percentage of effector $\mathrm{CD}^{+}$cells was observed only in the delayed-treatment group $(P<0.005$ as compared with controls) (Figure 6, a and b). These data clearly show that ICOS signaling is necessary for the generation and/or maintenance of alloreactive $\mathrm{CD}^{+}$effector $\mathrm{T}$ cells, and that this effect occurs after $\mathrm{T}$ cell priming.

The role of ICOS in alloantibody production. ICOS signaling plays a major role in immunoglobulin class switching $(14,17)$. To assess the role of ICOS-B7h on humoral alloimmune responses, we measured the levels of donorspecific alloantibody production by flow cytometry 10-14 days after transplantation in recipients treated with delayed anti-ICOS mAb. High levels of alloantibodies of IgG2a (Figure 6c) and IgG1 (Figure 6d) isotypes were observed in the sera of the untreated control recipients, which were significantly decreased after delayed ICOS blockade $(P<0.01$ as compared with control 

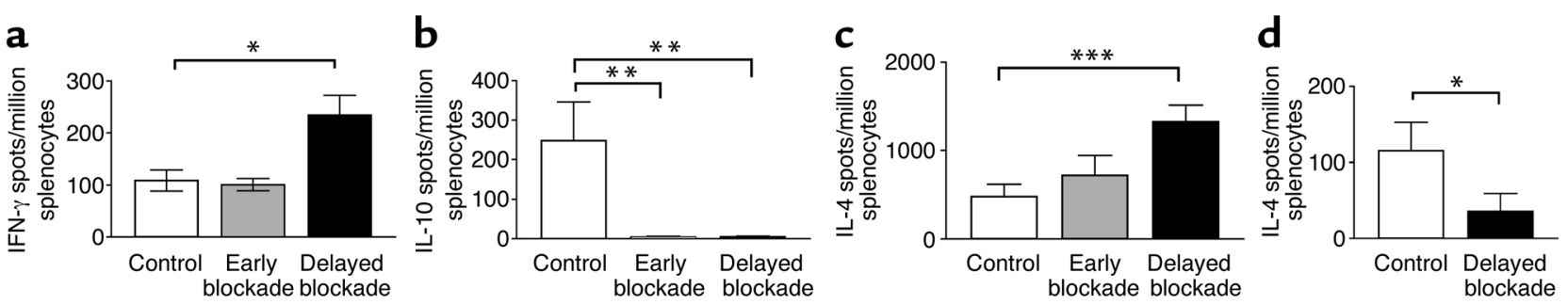

\section{Figure 4}

Frequency of alloantigen-specific cytokine-producing cells in recipients treated with early or delayed anti-ICOS mAb. BALB/c WT recipients transplanted with $\mathrm{C} 5 \mathrm{BL} / 6$ grafts were sacrificed 10 days after transplantation and their spleen cells used as responders, while irradiated spleen cells from naive C57BL/ 6 mice were used as stimulators. Data represent mean \pm SEM of triplicates from 3-5 animals per group. (a) IFN- $\gamma$. Early anti-ICOS mAb-treated recipients exhibited a similar frequency of alloreactive IFN- $\gamma$-producing $T$ cells as untreated recipients, while delayed anti-ICOS mAb-treated mice had significantly increased frequencies $(P=0.0115$ versus untreated control). (b) IL-10. Both early and delayed ICOS blockade resulted in significantly reduced frequencies of IL-10-producing alloreactive T cells $(P=0.0047$ for both early and delayed versus controls). (c) IL-4. Only delayed blockade produced a significant increase in the IL-4-producing frequencies ( $P=0.0006$ versus controls). (d) IL-4 production by splenocytes following ex vivo CD8 $8^{+}$depletion. There was a reduction in IL-4 frequencies following ex vivo $C D 8^{+} \mathrm{T}$ cell depletion using splenocytes from both controls and delayed ICOS blockade groups, although this was only significant in the animals treated with delayed ICOS blockade ( $P=0.0003$ for CD8-depleted versus nondepleted sample for delayed blockade). Moreover, there was a significant difference between the CD8-depleted samples with a lower IL-4 frequency in the delayed-treatment group ( $P=0.035$ for CD8-depleted control versus CD8-depleted delayed-blockade group), demonstrating a higher frequency of IL-4-producing cells in the presence of CD ${ }^{+}$T cells and following delayed ICOS blockade. ${ }^{*} P<0.05 ;{ }^{*} P<0.01 ;{ }^{*}{ }^{*} P<0.001$.

untreated animals) (Figure 6, c and d). Similar results were obtained with early blockade (data not shown). These data demonstrate the importance of ICOS signaling in the generation of class-switched alloantibodies.

The effect of ICOS blockade is dependent on $C D 8^{+} T$ cells. We then explored the effect of ICOS blockade on CD4 ${ }^{+}$ $\mathrm{T}$ cell-mediated rejection. Consistent with our previous results, transiently $\mathrm{CD}^{+} \mathrm{T}$ cell-depleted recipients demonstrated modest prolongation of allograft survival (8). In recipients treated with either early or late anti-ICOS $\mathrm{mAb}, \mathrm{CD}^{+} \mathrm{T}$ cell depletion completely abrogated the prolonged graft survival that ICOS blockade alone produced (Figure 7a). Moreover, a similar loss of graft prolongation was found when BALB $/ \mathrm{c}$ hearts were transplanted into CD8-deficient C57BL/6 mice (Figure $7 \mathrm{~b}$ ). These observations indicate that ICOS blockade cannot prevent solely $\mathrm{CD}^{+}{ }^{+} \mathrm{T}$ cellmediated rejection, which may be due to a lack of effect of ICOS blockade on $\mathrm{CD}^{+} \mathrm{T}$ cells or a lack of a population of $\mathrm{CD}^{+} \mathrm{T}$ regulatory cells (52-55). Given the data demonstrating significant inhibition of alloreactive $\mathrm{CD}^{+}$and effector $\mathrm{CD}^{+} \mathrm{T}$ cell expansion, as well as the cytokine profiles described above, these results demonstrate that the beneficial effects of ICOS blockade on graft survival are dependent on the presence of a population of regulatory $\mathrm{CD}^{+} \mathrm{T}$ cells.

\section{Figure 5}

The effect of delayed ICOS blockade in STAT4 ${ }^{-1-}$ and STAT6 ${ }^{-1-}$ recipients. Anti-ICOS mAb was used in BALB/c background STAT4 ${ }^{-1-}$ and STAT6 $^{-/-}$recipients transplanted with $\mathrm{C} 57 \mathrm{BL} / 6$ hearts. (a) As compared with the untreated STAT4 ${ }^{-/-}$controls (MST, 10 days; $n=4$ ), delayed ICOS blockade prolonged allograft survival in STAT4 ${ }^{-1}$ recipients (MST, 24 days; $n=5 ; P<0.005$ ). (b) By contrast, in STAT6 ${ }^{-/-}$ recipients, whereas untreated controls had similar graft survival (MST, 10 days; $n=4$ ), delayed ICOS blockade had no effect (MST, 10 days; $n=5$; not statistically significant as compared with untreated controls).
Interactions between the ICOS-B7h and CD28/CTLA-4-B7 pathways. In the absence of CD28 signaling, allografts can be rejected, albeit at a slower rate $(8,56)$. In part, this is thought to be due $T$ cell activation using alternative costimulatory pathways. Furthermore, there are complex interactions between ICOS-B7h and the CD28-B7 pathways. CD28 costimulation upregulates ICOS expression (18), whereas ligation of $\mathrm{B} 7 \mathrm{~h}$ results in downregulation of CD86 expression on APCs (57). Therefore, we investigated the effect of ICOS blockade in regulating alloimmune responses in the absence of CD28 costimulation. Untreated CD28deficient C57BL/ 6 recipients rejected their BALB/c grafts with an MST of 16 days. Both early (MST, 31 days) and delayed (MST, 70 days) ICOS blockade in

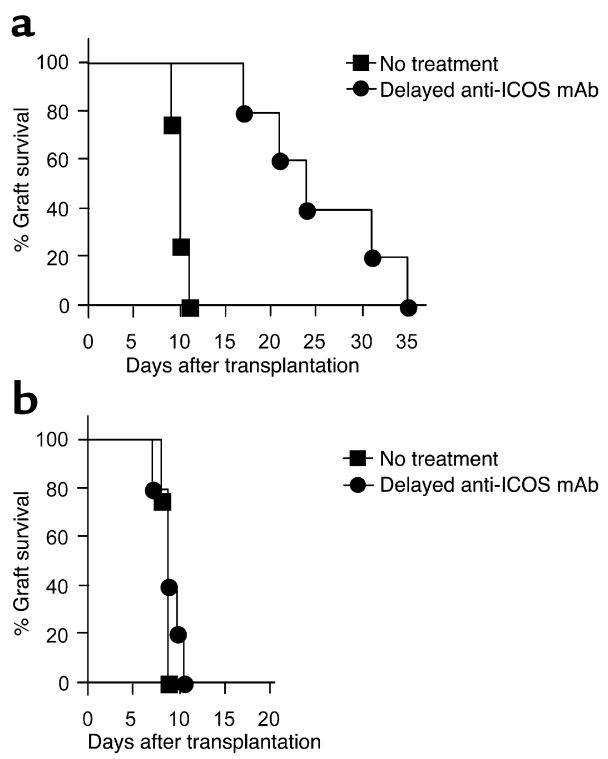


a
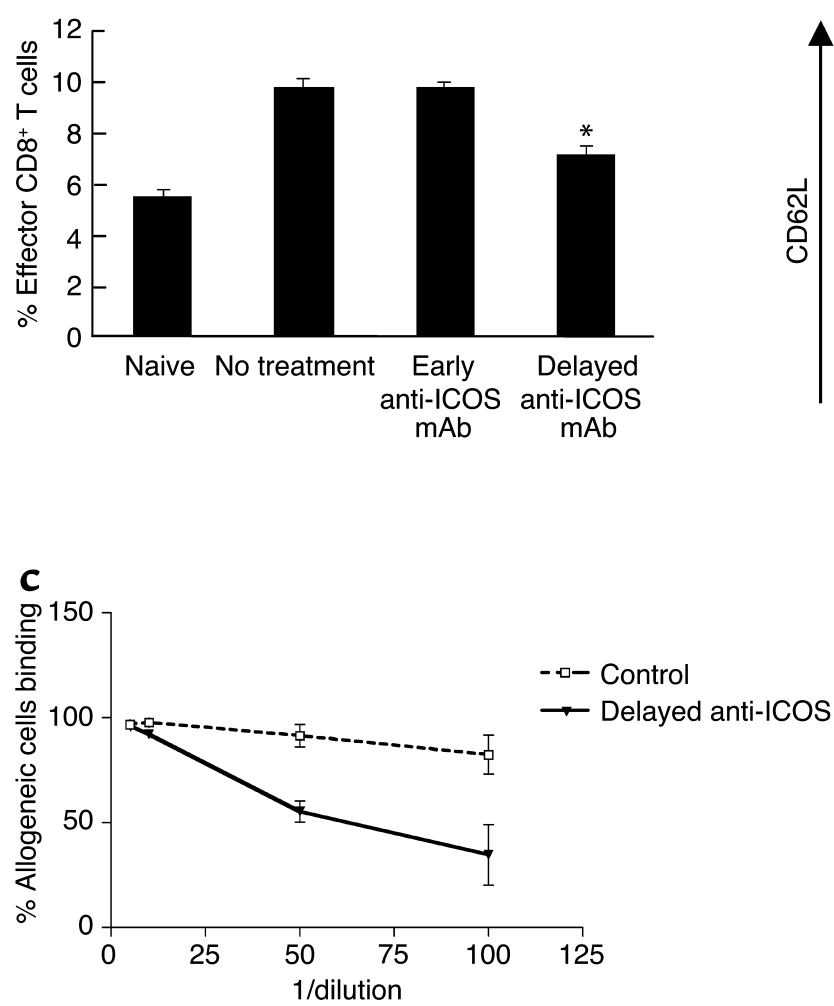

b

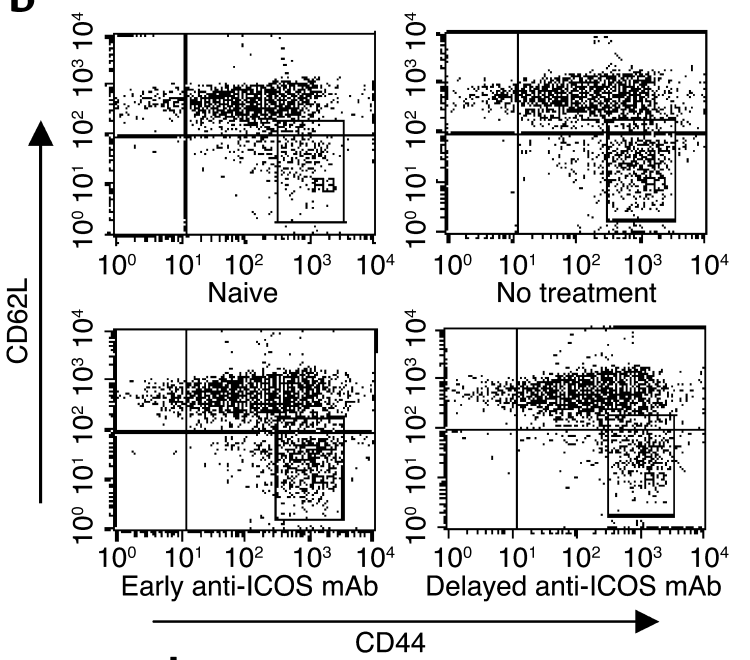

d

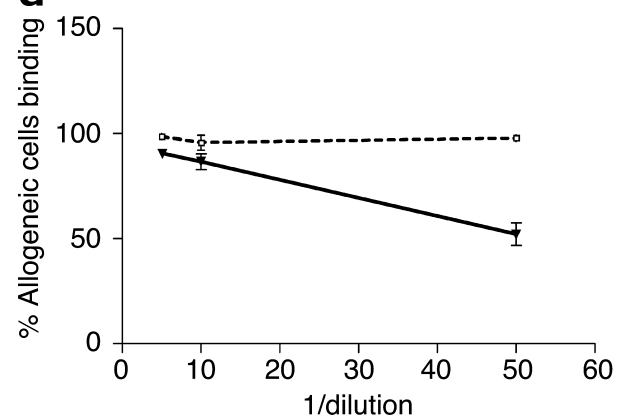

Figure 6

The effect of ICOS-B7h blockade on effector CD8 ${ }^{+} T$ cell generation and alloantibody production. (a and $\left.\mathbf{b}\right)$ Percentages of effector CD8 ${ }^{+}$ $T$ cells. Splenocytes obtained from transplant recipients were used for staining of effector $C D 8^{+} T$ cells, characterized as CD8 ${ }^{+} C D 44^{\text {high }}$ CDD62 Llow cells. The scatter plots are shown in (b). The population of effector CD8 ${ }^{+}$cells (gated in R3) in the early anti-ICOS treatment group is as high as that of the untreated controls (a). However, the number of effector CD8 ${ }^{+} \mathrm{T}$ cells was significantly suppressed in the delayed-treatment group. The representative data among three different experiments is expressed as the mean percentage \pm SEM, run in triplicates ( ${ }^{*} P<0.005$ as compared with the untreated control or the early anti-ICOS mAb treatment group). (c and d) Alloantibody production in allograft recipients treated with delayed ICOS-B7h blockade. Naive donor strain C57BL/6 splenocytes were incubated with serum from transplant recipients obtained 10 days after transplantation. Binding of alloantibody was assessed by flow cytometry analysis after incubation of FITC-conjugated rat anti-mouse IgG2a (c) or anti-mouse IgG1 (d) antibody. The percentage of donor cells binding at each serum dilution from three individual recipients per group is expressed. Production of alloantibody was observed in the sera of the untreated control recipients, whereas delayed anti-ICOS mAb treatment drastically inhibited the production of IgG1 and IgG2a alloantibodies $(P<0.01$ as compared with untreated controls). Similar reduction was seen in the early-treatment group (data not shown). $1 /$ dilution, reciprocal of dilution, i.e., $50=1$ in 50 dilution.

CD28-deficient recipients significantly prolonged graft survival $(P=0.0122$ for early blockade as compared with control, $P=0.0008$ for late blockade as compared with control) (Figure 8a). Thus, ICOS is an important regulator of alloimmune responses even in the absence of $\mathrm{CD} 28$ signaling.

ICOS expression is also regulated by CTLA-4 engagement (19). To investigate the effect of CTLA- 4 on regulating ICOS in alloimmune responses, we used a blocking anti-CTLA-4 mAb in combination with anti-ICOS $\mathrm{mAb}$ in both treatment protocols. Consistent with our previous data, CTLA- 4 blockade alone resulted in a small yet significant acceleration of acute allograft rejection (8). CTLA-4 blockade also promoted accelerated rejection with the concomitant use of ICOS blockade, regardless of which treatment protocol was used
(Figure 8, b and c). These data indicate that ICOS blockade is insufficient to overcome the loss of negative regulation after inhibition of CTLA-4 and that the beneficial effect on allograft survival is dependent on an intact CTLA-4 signaling pathway.

\section{Discussion}

The recently identified molecule ICOS (13), which is the third member of the CD28 superfamily, has some unique features separating it from CD28. ICOS costimulation results in the increased production of IL-4, IL-5, and IL-10 but not IL-2 $(13,18,58)$. ICOS, unlike CD28, is not constitutively expressed on the T cell surface but induced upon cell activation (13). The ICOS ligand B7h (20) is structurally related to the $\mathrm{B} 7$ molecules but binds neither CD28 nor CTLA- 4 . 

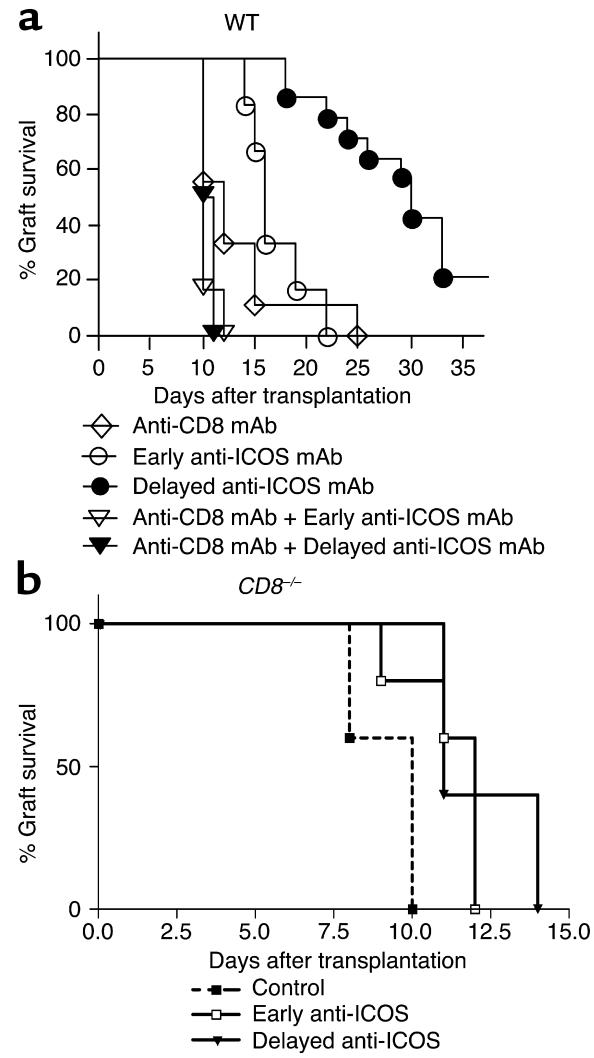

Likewise, ICOS binds neither CD80 nor CD86 (13). Thus, the ICOS-B7h pathway may provide a signal to compensate for a deficit in the CD28-CD80/ CD86 pathway or may play a unique role in addition to the $\mathrm{CD} 28$ pathway.
Figure 7

ICOS-B7h signal blockade in CD8-depleted or -deficient animals. Vascularized C57BL/ 6 hearts were transplanted into BALB/c recipients. (a) Anti-CD8-depleting mAb was administered to recipients preoperatively on days $-6,-3$, and -1 , whereas anti-ICOS $\mathrm{mAb}$ was used as above. Preoperative CD8 depletion modestly prolonged allograft survival as compared with untreated controls (MST, 12 days; $n=9 ; P<0.05$ ). Interestingly, the beneficial effects of antiICOS mAb on allograft survival were abrogated in both early- and delayed-treatment groups when $C D 8^{+} T$ cells were preoperatively depleted (MST, 10 and 10.5 days, respectively; $n=6 ; P<0.005$ and $P<0.0005$ as compared with early and delayed anti-ICOS mAb treatment groups, respectively). (b) Using CD8-deficient C57BL/6 recipients transplanted with $B A L B / c$ hearts, the beneficial effects of ICOS-B7h blockade using either treatment protocol were lost.

It has been previously demonstrated that blockade of the ICOS-B7h signal alone results in a modest prolongation of allograft survival $(30,59)$, and our results with early ICOS-B7h blockade confirmed these findings. The observation of allograft survival in $\mathrm{ICOS}^{-/-}$recipients further supports this notion. However, ICOS blockade at different stages of the immune response results in disparate outcomes in some autoimmune models, with early and late blockade exacerbating and ameliorating EAE, respectively $(33,34)$, and worsening acute but suppressing chronic graft-versus-host disease (35). Our data suggest that in solid organ transplantation, this dichotomous effect occurs only in the context of a smaller clone size of reacting $T$ cells. Both early and delayed ICOS blockade resulted in prolonged allograft survival in major-mismatched recipients with a larger frequency of alloreactive T cells, although delayed blockade does appear to be more effective. By contrast, in minor-mismatched recipients

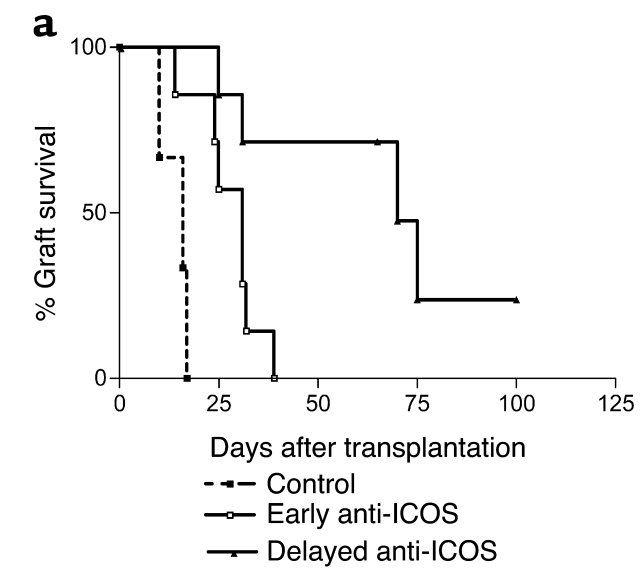

b

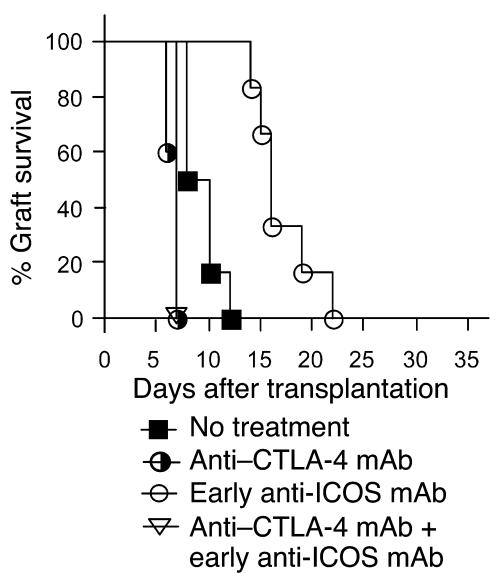

Figure 8

The interaction between the CD28/CTLA-4-B7 pathway and ICOS-B7h signal blockade. (a) CD28-deficient C57BL/6 recipients of a BALB/c heart all ultimately rejected their grafts (MST, 16 days). CD28-deficient recipients treated with early (MST, 31 days) or delayed (MST, 70 days) ICOS-B7h blockade had prolonged graft survival as compared with untreated controls $(P=0.0122$ for early treatment as compared with controls and $P=0.0008$ for delayed treatment as compared with controls), with delayed treatment resulting in significantly prolonged survival as compared with early blockade ( $P=0.0089$ for delayed as compared with early blockade). (b and $\mathbf{c})$ Anti-CTLA-4 mAb was used in conjunction with anti-ICOS mAb. Vascularized C57BL/ 6 hearts were transplanted into BALB/c recipients. Anti-CTLA-4 treatment alone accelerated allograft rejection (MST, 7 days; $n=4 ; P<0.005$ as compared with untreated control). Anti-CTLA-4 abrogated the prolongation of allograft survival in the recipients of early $(\mathbf{b})$ or delayed anti-ICOS mAb (c) treatment (MST, 7 and 6 days; $n=5 ; P<0.005$ and $P<0.0001$, respectively) as compared with recipients of early or delayed anti-ICOS mAb treatment. 
characterized by a smaller alloreactive $T$ cell clone size, early blockade accelerated graft rejection, while delayed blockade prolonged survival. However, T cell clone size is not the only factor determining the outcome of an immune response in the absence of ICOS signaling. Recent data using an autoimmune arthritis model demonstrated that, in contradiction to the EAE model, ICOS-deficient animals are protected from developing collagen-induced arthritis $(33,60)$.

ICOS blockade inhibited the effector mechanisms of allograft rejection, although that was dependent on the timing of antibody therapy. Alloantibody production was significantly suppressed in both early- and delayed-treatment groups and is in keeping with the recent data demonstrating an important role for ICOS in humoral immune responses (60). However, the effect on alloantibody production cannot alone explain the differences in allograft survival between the early- and delayed-blockade groups. Importantly, the production of $\mathrm{CD}^{+} \mathrm{T}$ cell effectors was significantly suppressed in the delayed-but not the early-treatment group, suggesting that the effect of ICOS blockade on effector $\mathrm{CD} 8^{+} \mathrm{T}$ cell generation in vivo is not secondary to inhibiting early $\mathrm{CD}^{+} \mathrm{T}$ cell help. These data are in keeping with an important role for ICOS-B7h signaling in enhancing both naive and recall $\mathrm{CD}^{+} \mathrm{T}$ cell responses $(50,51)$. Taken together, these data indicate that the contribution of ICOS costimulation to $\mathrm{T}$ cell responses and the functional consequences of ICOS blockade may be critically influenced by both the nature of the immune response, the responding $\mathrm{T}$ cell clone size, and the timing of intervention (61).

ICOS-B7h signaling plays a role in both Th1 and Th2 differentiation (29-32), although it appears to be more crucial for Th2 development. However, the precise effect of ICOS deficiency or blockade on antigen-specific cytokine responses appears in part to depend on the models studied and the timing of blockade $(30,33,60$, 62). The ICOS signal is necessary for Th2 cell development after immune priming, so that early blockade may result in a skewing to a Th1 response, and delayed blockade during the effector phase would maintain a Th2 response. Our data demonstrate that the frequency of alloreactive IL-10-producing recipient spleen cells with early or delayed anti-ICOS mAb treatment was significantly lower than in the control group, in keeping with findings from an allergen-induced airway hyperreactivity model (62), whereas the frequency of IFN- $\gamma$-producing cells was higher in the delayed-treatment group. However, we demonstrated that in the delayed-treatment group there was a significant increase in IL-4 production, which was dependent on the presence of $\mathrm{CD} 8^{+} \mathrm{T}$ cells (there was also an increase in the early-treatment group but this was not statistically significant). Dissociation between IL-4 and IL-10 production after ICOS blockade has been previously reported (62). Interestingly, we found that ICOS blockade was ineffective in prolonging graft survival in the absence of $\mathrm{CD}^{+} \mathrm{T}$ cells. Data from our alloreactive TCR transgenic model indicate that this is not due to a lack of effect on the $\mathrm{CD}^{+} \mathrm{T}$ cell population, whose expansion in vivo was significantly reduced after ICOS blockade. These data suggest that a subset of $\mathrm{CD}^{+} \mathrm{T}$ cells may be regulatory in nature. The mechanism by which the $\mathrm{CD}^{+} \mathrm{T}$ cells regulate remains uncertain. However, significant loss of IL-4 production after ex-vivo $\mathrm{CD}^{+} \mathrm{T}$ cell depletion suggests that these cells either directly or indirectly produce IL-4. Furthermore, and confirming these findings, our studies in STAT4 ${ }^{-/-}$and $S T A T 6^{-/}$mice demonstrated that delayed ICOS blockade was not effective in STAT6-deficient recipients that are also deficient in Th2 cytokines. The importance and function of CD8 $8^{+}$ regulatory $T$ cells have been recently appreciated (52-55). A number of mechanisms of action have been found for such cells using different models, including a direct effect on pathogenic $\mathrm{CD} 4^{+}$Th cell phenotype (63) and an effect mediated through alterations in APC function (54). Definitive proof of a role for regulatory $\mathrm{CD}^{+} \mathrm{T}$ cell populations in allotransplantation after ICOS blockade and their characterization and mechanisms of action require further in-depth studies.

Although there are a number of costimulatory molecules capable of fully activating T cells, the various expression patterns and resultant effector functions of these molecules suggest some degree of compartmentalization or hierarchy among the costimulatory pathways $(64,65)$. Interestingly, despite CD28 costimulation optimizing ICOS expression, we have shown that in the absence of CD28 signaling ICOS remains an important mechanism for $\mathrm{T}$ cell activation during alloimmune responses, in part explaining how grafts are rejected despite an absence of CD28 costimulation. Furthermore, the beneficial effects of ICOS blockade are lost in the absence of CTLA- 4 signaling. These findings further demonstrate the complex interactions between these two pathways in vivo.

In conclusion, we have shown that ICOS-B7h blockade induced prolongation of allograft survival more effectively during the effector/differentiation phase than the priming phase of the alloimmune response and that ICOS-B7h signaling is important for $\mathrm{T}$ helper cell differentiation in alloimmunity. Therefore, given our results, manipulation of the ICOS-B7h signaling pathway in order to prolong graft survival may be best initiated after $T$ cell priming.

\section{Acknowledgments}

We thank Christopher S. Geehan, Karla S. Stenger, and Susan P. Shea for their invaluable technical assistance. This work was supported by NIH grants 1RO1 AI51559 and 5 PO1 AI41521 (to M.H. Sayegh) and CA84500 and P01 AI39671 (to G.J. Freeman).

\footnotetext{
1. Sayegh, M.H., and Turka, L.A. 1998. The role of T-cell costimulatory activation pathways in transplant rejection. N. Engl. J. Med. 338:1813-1821.

2. Salomon, B., and Bluestone, J.A. 2001. Complexities of CD28/B7: CTLA-4 costimulatory pathways in autoimmunity and transplantation. Annu. Rev. Immunol. 19:225-252.

3. Coyle, A.J., and Gutierrez-Ramos, J.C. 2001. The expanding B7 superfamily: increasing complexity in costimulatory signals regulating $\mathrm{T}$ cell function. Nat. Immunol. 2:203-209.

4. Hancock, W.W., et al. 1996. Costimulatory function and expression of CD40 ligand, CD80, and CD86 in vascularized murine cardiac allograft rejection. Proc. Natl. Acad. Sci. U. S. A. 93:13967-13972.
} 
5. Grewal, I.S., and Flavell, R.A. 1998. CD40 and CD154 in cell-mediated immunity. Annu. Rev. Immunol. 16:111-135.

6. Larsen, C.P., et al. 1996. Long-term acceptance of skin and cardiac allografts after blocking CD40 and CD28 pathways. Nature. 381:434-438. 7. Lin, H., et al. 1998. Cytotoxic T lymphocyte antigen 4 (CTLA-4) blockade accelerates the acute rejection of cardiac allografts in CD28-deficient mice: CTLA-4 can function independently of CD28. J. Exp. Med. 188:199-204.

8. Yamada, A., et al. 2001. CD28-independent costimulation of T cells in alloimmune responses. J. Immunol. 167:140-146.

9. Shimizu, K., Schonbeck, U., Mach, F., Libby, P., and Mitchell, R.N. 2000 Host CD40 ligand deficiency induces long-term allograft survival and donor-specific tolerance in mouse cardiac transplantation but does not prevent graft arteriosclerosis. J. Immunol. 165:3506-3518.

10. London, C.A., Lodge, M.P., and Abbas, A.K. 2000. Functional responses and costimulator dependence of memory CD4+ T cells. J. Immunol. 164:265-272.

11. Valujskikh, A., Pantenburg, B., and Heeger, P.S. 2002. Primed allospecific $T$ cells prevent the effects of costimulatory blockade on prolonged cardiac allograft survival in mice. Am. J. Transplant. 2:501-509.

12. Ensminger, S.M., et al. 2000. CD8+ T cells contribute to the development of transplant arteriosclerosis despite CD154 blockade. Transplantation. 69:2609-2612

13. Hutloff, A., et al. 1999. ICOS is an inducible T-cell co-stimulator structurally and functionally related to CD28. Nature. 397:263-266.

14. McAdam, A.J., et al. 2001. ICOS is critical for CD40-mediated antibody class switching. Nature. 409:102-105.

15. Dong, C., et al. 2001. ICOS co-stimulator receptor is essential for T-cell activation and function. Nature. 409:97-101.

16. Dong, C., Temann, U.A., and Flavell, R.A. 2001. Cutting edge: critical role of inducible costimulator in germinal center reactions. J. Immunol. 166:3659-3662.

17. Tafuri, A., et al. 2001. ICOS is essential for effective T-helper-cell responses. Nature. 409:105-109.

18. McAdam, A.J., et al. 2000. Mouse inducible costimulatory molecule (ICOS) expression is enhanced by CD28 costimulation and regulates differentiation of CD4+ T cells. J. Immunol. 165:5035-5040.

19. Riley, J.L., et al. 2001. ICOS costimulation requires IL-2 and can be prevented by CTLA-4 engagement. J. Immunol. 166:4943-4948.

20. Swallow, M.M., Wallin, J.J., and Sha, W.C. 1999. B7h, a novel costimulatory homolog of B7.1 and B7.2, is induced by TNFalpha. Immunity. 11:423-432.

21. Yoshinaga, S.K., et al. 1999. T-cell co-stimulation through B7RP-1 and ICOS. Nature. 402:827-832.

22. Aicher, A., et al. 2000. Characterization of human inducible costimulator ligand expression and function. J. Immunol. 164:4689-4696.

23. Brodie, D., et al. 2000. LICOS, a primordial costimulatory ligand? Curr. Biol. 10:333-336.

24. Ling, V., et al. 2000. Cutting edge: identification of GL50, a novel B7-like protein that functionally binds to ICOS receptor. J. Immunol. 164:1653-1657.

25. Gonzalo, J.A., et al. 2001. Cutting edge: the related molecules CD28 and inducible costimulator deliver both unique and complementary signals required for optimal $\mathrm{T}$ cell activation. J. Immunol. 166:1-5.

26. Coyle, A.J., et al. 2000. The CD28-related molecule ICOS is required for effective T cell-dependent immune responses. Immunity. 13:95-105.

27. Sporici, R.A., and Perrin, P.J. 2001. Costimulation of memory T-cells by ICOS: a potential therapeutic target for autoimmunity? Clin. Immunol. 100:263-269.

28. Sperling, A.I., and Bluestone, J.A. 2001. ICOS costimulation: it's not just for TH2 cells anymore. Nat. Immunol. 2:573-574.

29. Greenwald, R.J., McAdam, A.J., Van Der Woude, D., Satoskar, A.R., and Sharpe, A.H. 2002. Cutting edge: inducible costimulator protein regulates both Th1 and Th2 responses to cutaneous leishmaniasis. J. Immunol. 168:991-995.

30. Ozkaynak, E., et al. 2001. Importance of ICOS-B7RP-1 costimulation in acute and chronic allograft rejection. Nat. Immunol. 2:591-596.

31. Carreno, B.M., and Collins, M. 2002. The B7 family of ligands and its receptors: new pathways for costimulation and inhibition of immune responses. Annu. Rev. Immunol. 20:29-53.

32. Gonzalo, J.A., et al. 2001. ICOS is critical for T helper cell-mediated lung mucosal inflammatory responses. Nat. Immunol. 2:597-604.

33. Rottman, J.B., et al. 2001. The costimulatory molecule ICOS plays an important role in the immunopathogenesis of EAE. Nat. Immunol. 2:605-611.

34. Sporici, R.A., et al. 2001. ICOS ligand costimulation is required for T-cell encephalitogenicity. Clin. Immunol. 100:277-288.

35. Ogawa, S., et al. 2001. Opposing effects of anti-activation-inducible lymphocyte- immunomodulatory molecule/inducible costimulator antibody on the development of acute versus chronic graft-versus-host disease. J. Immunol. 167:5741-5748.

36. Iwai, H., et al. 2002. Amelioration of collagen-induced arthritis by blockade of ICOS-B7h costimulation. J. Immunol. 169:4332-4339.
37. Walunas, T.L., et al. 1994. CTLA-4 can function as a negative regulator of $\mathrm{T}$ cell activation. Immunity. 1:405-413.

38. Corry, R.J., Winn, H.J., and Russell, P.S. 1973. Primarily vascularized allografts of hearts in mice. The role of $\mathrm{H}-2 \mathrm{D}, \mathrm{H}-2 \mathrm{~K}$, and non-H-2 antigens in rejection. Transplantation. 16:343-350.

39. Matesic, D., Lehmann, P.V., and Heeger, P.S. 1998. High-resolution characterization of cytokine-producing alloreactivity in naive and allograftprimed mice. Transplantation. 65:906-914.

40. Kishimoto, K., et al. 2000. The role of CD154-CD40 versus CD28-B7 costimulatory pathways in regulating allogeneic Th1 and Th2 responses in vivo. J. Clin. Invest. 106:63-72.

41. Sho, M., et al. 2002. New insights into the interactions between T cell costimulatory blockade and conventional immunosuppressive drugs. Ann. Surg. 236:667-675.

42. Zhai, Y., et al. 2002. The CD154-CD40 $\mathrm{T}$ cell costimulation pathway is required for host sensitization of $\mathrm{CD} 8(+) \mathrm{T}$ cells by skin grafts via direct antigen presentation. J. Immunol. 169:1270-1276.

43. Marsh, J.E., et al. 2001. The allogeneic T and B cell response is strongly dependent on complement components C3 and C4. Transplantation. 72:1310-1318

44. Backstrom, B.T., Muller, U., Hausmann, B., and Palmer, E. 1998. Positive selection through a motif in the alphabeta $\mathrm{T}$ cell receptor. Science. 281:835-838.

45. Sayegh, M.H., et al. 2003. Allograft rejection in a new allospecific CD4+ TCR transgenic mouse. Am. J. Transplant. 3:381-389.

46. Sandner, S.E., et al. 2003. The first model of tracking allospecific CD4+ $\mathrm{T}$ cell activation and tolerance in vivo. Am. J. Transplant. In press.

47. Suchin, E.J., et al. 2001. Quantifying the frequency of alloreactive T cells in vivo: new answers to an old question. J. Immunol. 166:973-981.

48. Sho, M., et al. 2002. Physiological mechanisms of regulating alloimmunity: cytokines, CTLA-4, CD25+ cells, and the alloreactive T cell clone size. J. Immunol. 169:3744-3751.

49. Kopf, M., et al. 2000. Inducible costimulator protein (ICOS) controls T helper cell subset polarization after virus and parasite infection. J. Exp. Med. 192:53-61.

50. Wallin, J.J., Liang, L., Bakardjiev, A., and Sha, W.C. 2001. Enhancement of CD8+ T cell responses by ICOS/B7h costimulation. J. Immunol. 167:132-139.

51. Liu, X., et al. 2001. B7H costimulates clonal expansion of, and cognate destruction of tumor cells by, CD8(+) T lymphocytes in vivo. J. Exp. Med. 194:1339-1348

52. Jones, H.P., Tabor, L., Sun, X., Woolard, M.D., and Simecka, J.W. 2002 Depletion of CD8 $+\mathrm{T}$ cells exacerbates CD4+ Th cell-associated inflammatory lesions during murine mycoplasma respiratory disease. J. Immunol. 168:3493-3501.

53. Gilliet, M., and Liu, Y.J. 2002. Generation of human CD8 T regulatory cells by CD40 ligand-activated plasmacytoid dendritic cells. J. Exp. Med. 195:695-704.

54. Chang, C.C., et al. 2002. Tolerization of dendritic cells by T(S) cells: the crucial role of inhibitory receptors ILT3 and ILT4. Nat. Immunol. 3:237-243.

55. Colovai, A.I., et al. 2003. Regulatory CD8+CD28- T cells in heart transplant recipients. Hum. Immunol. 64:31-37.

56. Lin, H., et al. 1998. Cytotoxic T lymphocyte antigen 4 (CTLA-4) blockade accelerates the acute rejection of cardiac allografts in CD28-deficient mice: CTLA-4 can function independently of CD28. J. Exp. Med. 188:199-204.

57. Salama, A.D., et al. 2003. The interaction between ICOS-B7RP1 and CD28-B7 costimulatory pathways in alloimmune responses in vivo. Am. J. Transplant. 3:390-395.

58. Guo, J., et al. 2001. Stimulatory effects of B7-related protein-1 on cellular and humoral immune responses in mice. J. Immunol. 166:5578-5584.

59. Guo, L., et al. 2002. Prolonged survival in rat liver transplantation with mouse monoclonal antibody against an inducible costimulator (ICOS). Transplantation. 73:1027-1032.

60. Nurieva, R.I., Treuting, P., Duong, J., Flavell, R.A., and Dong, C. 2003. Inducible costimulator is essential for collagen-induced arthritis. J. Clin Invest. 111:701-706. doi:10.1172/JCI200317321.

61. Greenwald, R.J., Latchman, Y.E., and Sharpe, A.H. 2002. Negative coreceptors on lymphocytes. Curr. Opin. Immunol. 14:391-396.

62. Akbari, O., et al. 2002. Antigen-specific regulatory T cells develop via the ICOS-ICOS-ligand pathway and inhibit allergen-induced airway hyperreactivity. Nat. Med. 8:1024-1032.

63. Jiang, H., Braunstein, N.S., Yu, B., Winchester, R., and Chess, L. 2001. CD8+ T cells control the TH phenotype of MBP-reactive CD4+ T cells in EAE mice. Proc. Natl. Acad. Sci. U. S. A. 98:6301-6306.

64. Yamada, A., Salama, A.D., and Sayegh, M.H. 2002. The role of novel $\mathrm{T}$ cell costimulatory pathways in autoimmunity and transplantation. J. Am. Soc. Nephrol. 13:559-575.

65. Salama, A.D., and Sayegh, M.H. 2003. Alternative T-cell costimulatory pathways in transplant rejection and tolerance induction: hierarchy or redundancy? Am. J. Transplant. 3:509-511. 